PROCEEDINGS OF THE

AMERICAN MATHEMATICAL SOCIETY

Volume 134, Number 11, November 2006, Pages 3123-3130

S 0002-9939(06)08383-3

Article electronically published on May 12, 2006

\title{
FIXED POINT SPACES, PRIMITIVE CHARACTER DEGREES AND CONJUGACY CLASS SIZES
}

\author{
I. M. ISAACS, THOMAS MICHAEL KELLER, U. MEIERFRANKENFELD, \\ AND ALEXANDER MORETÓ
}

(Communicated by Jonathan I. Hall)

\begin{abstract}
Let $G$ be a finite group that acts on a nonzero finite dimensional vector space $V$ over an arbitrary field. Assume that $V$ is completely reducible as a $G$-module, and that $G$ fixes no nonzero vector of $V$. We show that some element $g \in G$ has a small fixed-point space in $V$. Specifically, we prove that we can choose $g$ so that $\operatorname{dim} \mathbf{C}_{V}(g) \leq(1 / p) \operatorname{dim} V$, where $p$ is the smallest prime divisor of $|G|$.
\end{abstract}

\section{INTRODUCTION}

Let $V$ be a nontrivial finite-dimensional $F G$-module, where $F$ is a field and $G$ is a finite group. In his Ph.D. thesis [5], P. M. Neumann proved that if $G$ is solvable and $V$ is irreducible and nontrivial, then there exists an element of $G$ with small fixed-point subspace. Specifically, he showed that there exists $g \in G$ such that

$$
\operatorname{dim} \mathbf{C}_{V}(g) \leq \frac{7}{18} \operatorname{dim} V .
$$

Neumann also conjectured that in fact, there should exist $g \in G$ such that $\operatorname{dim} \mathbf{C}_{V}(g)$ $\leq(1 / 3) \operatorname{dim} V$.

One of the goals of this note is to prove results like Neumann's, but with milder assumptions on $G$ and $V$. In particular, we drop the assumption that $G$ is solvable and we weaken the condition that $V$ is irreducible. Clearly, however, some assumption on $V$ is essential since, for example, if $V$ has some huge direct summand on which $G$ acts trivially, then the ratio $\operatorname{dim} \mathbf{C}_{V}(g) / \operatorname{dim} V$ can be arbitrarily close to 1 for all elements $g \in G$. But even if $\mathbf{C}_{V}(G)$ is trivial, it is possible that no element of $G$ has a small fixed-point space in $V$. For example, let $V$ be the $n$-dimensional row space over an arbitrary field $F$ of characteristic different from 2, and let $G$ be the group of $n \times n$ matrices over $F$ with (1,1)-entry equal to \pm 1 , with all other diagonal entries equal to 1 and with $(i, j)$ entry equal to 0 if $j>1$ and $j \neq i$. Then in the natural action by right multiplication of $G$ on $V$, it is easy to check that $\mathbf{C}_{V}(G)=0$ and that every nonidentity element of $G$ centralizes a hyperplane in $V$.

Received by the editors June 2, 2005.

2000 Mathematics Subject Classification. Primary 20 C99.

The fourth author was partially supported by the Spanish Ministerio de Educación y Ciencia, grants MTM2004-04665 and MTM2004-06067-C02-01, the FEDER and the Programa Ramón y Cajal.

(C)2006 American Mathematical Society Reverts to public domain 28 years from publication 
We can obtain inequalities of the desired type, however, if we assume that $V$ is completely reducible. Our main result is the following.

Theorem A. Let $V$ be a nonzero finite-dimensional completely reducible $F G$ module, where $F$ is any field and $G$ is any finite group. Assume that $\mathbf{C}_{V}(G)=0$ and let $p$ be the smallest prime divisor of $|G|$. Then there exists some element $g \in G$ such that

$$
\operatorname{dim} \mathbf{C}_{V}(g) \leq \frac{1}{p} \operatorname{dim} V .
$$

The fraction $1 / p$ cannot, in general, be replaced by any smaller quantity.

In particular, this shows that Neumann's conjecture is valid for odd-order groups, at least, and without his assumption that the module is irreducible. We mention that our proof of Theorem A in the case where $G$ is nonsolvable relies on the classification of simple groups.

It also seems natural to look at the average dimension of the fixed-point spaces. As pointed out in [6], one can show, by looking at the character that corresponds to the module $V$, that if $V$ is nonprincipal and irreducible and $(|G|,|V|)=1$, then

$$
\frac{1}{|G|} \sum_{g \in G} \operatorname{dim} \mathbf{C}_{V}(g) \leq \frac{1}{2} \operatorname{dim} V .
$$

It seems reasonable to hope that this also holds if $|G|$ and $|V|$ are not coprime, and indeed, this was proved for solvable groups in Theorem 6.1 of $[6$. Proposition 2.1 of 7] proves a weaker inequality of this type for arbitrary groups $G$ and nonprincipal irreducible modules $V$ :

$$
\frac{1}{|G|} \sum_{g \in G} \operatorname{dim} \mathbf{C}_{V}(g) \leq \frac{3}{4} \operatorname{dim} V .
$$

As a consequence of our Theorem A, we can obtain this inequality with a weaker assumption on $V$.

Corollary B. Let $V$ be a nonzero finite-dimensional completely reducible FGmodule, where $F$ is an arbitrary field and $G$ is any finite group, and assume that $\mathbf{C}_{V}(G)=0$. Then

$$
\frac{1}{|G|} \sum_{g \in G} \operatorname{dim} \mathbf{C}_{V}(g) \leq \frac{p+1}{2 p} \operatorname{dim} V,
$$

where $p$ is the smallest prime divisor of $|G|$.

In particular, in the situation of Corollary $\mathrm{B}$, the average fixed-point-space dimension can never exceed $(3 / 4) \operatorname{dim} V$. Although we have no reason to believe that the coefficient $3 / 4$ is best possible here, we will see that in general, it cannot be replaced by any number smaller than $1 / 2$.

We now digress to explain our original motivation for considering these questions. There are numerous parallels and analogies between theorems concerning the set of irreducible character degrees of a finite group and theorems concerning the set of conjugacy class sizes of such groups. This suggests that perhaps there are some subtle arithmetic connections between these two sets of integers associated with a given group. One such connection that is easy to see is that each prime number 
that divides an irreducible character degree of $G$ must also divide some class size of $G$. If $G$ is solvable, then S. Dolfi showed that more is true. He proved [2] that given any two distinct primes $p$ and $q$ such that $p q$ divides some irreducible character degree of a solvable group $G$, then $p q$ also divides some class size of $G$. One might conjecture that the analogous assertion for three or more distinct primes is also true, but as far as we know, this remains open.

Could it be true that each irreducible character degree of a finite group $G$ divides some class size of $G$ ? Or failing that, we could ask if each irreducible character degree of $G$ divides the $n$th power of some class size for some fixed integer $n$. As extraspecial $p$-groups show, however, no such assertion can be true in general. But what if we insist that the irreducible character is primitive? We know no counterexample to the following.

Conjecture C. Let $\chi$ be a primitive irreducible character of an arbitrary finite group $G$. Then $\chi(1)$ divides $\left|\mathrm{cl}_{G}(g)\right|$ for some element $g \in G$.

Here, of course, we have written $\mathrm{cl}_{G}(g)$ to denote the class of $g$ in $G$. We have checked that Conjecture $\mathrm{C}$ holds for all irreducible characters (primitive or not) of all groups in the Atlas [1].

As a consequence of our fixed-point-spaces theorem, we can work one prime at a time to obtain a weak result in the direction of Conjecture C. In the following, we use the notation $n_{p}$ to denote the $p$ part of a positive integer $n$, where $p$ is a prime number.

Corollary D. Let $\chi$ be a primitive irreducible character of a solvable group $G$, and let $p$ be a prime divisor of $|G|$. Then $\chi(1)_{p}$ divides $\left(\left|\mathrm{cl}_{G}(g)\right|_{p}\right)^{3}$ for some element $g \in G$.

Even though this result is quite weak, it shows that imposing the primitivity assumption makes Conjecture C plausible.

We thank J. Sangroniz for helpful comments on an earlier draft of this paper. Much of this work was done while the second author was on sabbatical leave and both he and the fourth author were visiting the University of Wisconsin, Madison. They thank the Mathematics Department there for its hospitality.

\section{Centralizer Dimensions}

In this section, we prove Theorem A. In fact, we establish a strong form of this result. We show that an element with small fixed-point space can be chosen in a specified coset of an appropriate normal subgroup. To recover the inequality of Theorem A, we simply take $K=G$ in the following. (We will later show that the fraction $1 / p$ in Theorem A is best possible.)

Theorem 2.1. Let $V$ be a nonzero $F G$-module, where $G$ is an arbitrary finite group, and let $K$ be a normal subgroup of $G$. Assume that $\mathbf{C}_{V}(K)=0$ and that $V$ is completely reducible as an $F K$-module. Then each coset of $K$ in $G$ contains an element $t$ such that

$$
\operatorname{dim} \mathbf{C}_{V}(t) \leq \frac{1}{p} \operatorname{dim} V,
$$

where $p$ is the smallest prime divisor of $|G|$.

In order to handle the case where $K$ is nonsolvable in Theorem 2.1 we will use the following result of Guralnick and Kantor, which is stated as a corollary 
in Section 1 of [3]. Of course, the proof of this fact relies on the simple group classification.

Lemma 2.2. Let $G$ be a finite group whose socle $S$ is nonabelian and simple, and let $g \in G$ be an arbitrary nonidentity element. Then there exists an element $h \in G$ such that $S \subseteq\langle g, h\rangle$.

Actually, we need a very slight refinement of this lemma.

Corollary 2.3. In the situation of the foregoing lemma, the element $h$ can be selected from the subgroup $S$.

Proof. Since $\mathbf{C}_{G}(S)=1$, we see that $S$ is also the socle of the subgroup $S\langle g\rangle$, and so it is no loss to assume that $G=S\langle g\rangle$. Now choose $h$ by Lemma 2.2 and write $h=s g^{n}$ for some element $s \in S$ and integer $n \geq 0$. Then $\langle g, s\rangle \supseteq\langle g, h\rangle \supseteq S$, as required. 2.3 .

The key to our proof of Theorem 2.1 is the following application of Corollary

Theorem 2.4. Let $N$ be a minimal normal subgroup of a finite group $G$, and suppose that $G / N$ is cyclic with generating coset $N t$. Then there exist elements $x, y \in N$ such that $G=\langle x, y\rangle$.

Proof. First, suppose that $N$ is abelian, and let $t$ be any element of the given generating coset. Choose an arbitrary nonidentity element $n \in N$ and let $H=$ $\langle n t, t\rangle=\langle n, t\rangle$, so that $N \cap H>1$. Now $N \cap H$ is normalized by $H$, and because we are assuming that $N$ is abelian, $N \cap H$ is also normalized by $N$. Since $N H \supseteq$ $N\langle t\rangle=G$, it follows that $N \cap H \triangleleft G$. As $N$ is minimal normal in $G$, we have $N \cap H=N$, so $N \subseteq H$ and $G=N H=H$, as wanted.

We can now assume that $N=S_{1} \times \cdots \times S_{n}$ is a direct product of $n \geq 1$ nonabelian simple subgroups $S_{i}$. These factors are transitively permuted by $\langle t\rangle$, and so $N\left\langle t^{n}\right\rangle$ is the normalizer in $G$ of each of the subgroups $S_{i}$. If $t^{n}$ centralizes $S_{1}$, we argue that $(u t)^{n}$ does not centralize $S_{1}$, where $u \in S_{1}$ is an arbitrary nonidentity element. To see this, observe that $(u t)^{n}$ has the form $u_{1} u_{2} \cdots u_{n} t^{n}$, where $u_{i}$ is a conjugate of $u$ lying in $S_{i}$. We are assuming that $t^{n}$ centralizes $S_{1}$, and we know that each of the factors $u_{i}$ with $i>1$ also centralizes $S_{1}$, and thus since $u_{1}$ does not centralize $S_{1}$, it follows that $(u t)^{n}$ does not centralize $S_{1}$, as claimed. We can thus replace $t$ by $u t$ and assume that $t^{n}$ does not centralize $S_{1}$.

Now for notational simplicity, write $S=S_{1}$ and $z=t^{n}$, and let $M=N\langle z\rangle$, so that $M=\mathbf{N}_{G}(S)$. Let $C=\mathbf{C}_{G}(S)$ and observe that $S C / C$ is a simple normal subgroup of $M / C$. In fact, $S C / C$ is the unique minimal normal subgroup of $M / C$ since if $K / C$ is any other minimal normal subgroup, then $[S, K] \subseteq C$. But also $[S, K] \subseteq S$ since $S \triangleleft M$, and thus $[S, K] \subseteq S \cap C=1$. Then $K \subseteq \mathbf{C}_{G}(S)=C$, which is not the case.

The group $\bar{M}=M / C$ has the simple socle $\bar{S}$, and we know that $\bar{z}$ is a nonidentity element of $\bar{M}$. By Corollary 2.3 there exists a necessarily nonidentity element $\bar{s} \in \bar{S}$ such that $\langle\bar{z}, \bar{s}\rangle$ contains $\bar{S}$. Let $H=\langle z, s\rangle$, where $s$ is any preimage of $\bar{s}$ in $S$.

Now $\bar{S} \subseteq\langle\bar{z}, \bar{s}\rangle=\bar{H}$, so $S \subseteq H C$, and in fact, $S \triangleleft H C$ since $H C \subseteq M$. As both $H$ and $C=\mathbf{C}_{G}(S)$ normalize $H \cap S$, it follows that $H \cap S \triangleleft S$, and thus $H \cap S=S$, since $S$ is simple and $H \cap S$ contains $s$, and so is nontrivial. Then $S \subseteq H=\langle s, z\rangle \subseteq\langle s, t\rangle$, and thus $N \subseteq\langle s, t\rangle$ since each of the factors $S_{i}$ of $N$ is 
conjugate to $S$ via an element of $\langle t\rangle$. We now have $G=N\langle t\rangle \subseteq\langle s, t\rangle=\langle s t$, $t\rangle$, as required.

Proof of Theorem 2.1. We proceed by induction on $\operatorname{dim} V$, and we assume (as we may) that $K$ acts faithfully on $V$ and that $G=K\langle t\rangle$, where $K t$ is the given coset of $K$. By hypothesis, $\mathbf{C}_{V}(K)=0$, and hence $K>1$, and we can choose $N \subseteq K$ so that $N$ is minimal normal in $G$. Also, since $V$ is completely reducible as an $F K$ module and $N \triangleleft K$, Clifford's theorem guarantees that $V$ is completely reducible as an $F N$-module. Thus if $\mathbf{C}_{V}(N)=0$, we can replace $K$ by $N$ and assume that $K$ is minimal normal in $G$.

Otherwise, write $U=\mathbf{C}_{V}(N)>0$, and observe that $U<V$ and that $U$ admits the action of $G$. By complete reducibility, $U$ has an $N$-invariant complement $W$ in $V$, and since $N$ acts trivially on $U$, it follows that $[V, N] \subseteq W$. Also $[V, N]$ has an $N$-invariant complement in $V$, and we see that $N$ must centralize this complement. Then $V=[V, N]+U$, and it follows that $[V, N]=W$. In particular, we conclude that $W$ admits the action of $G$, and thus $V$ is the direct sum of proper $F G$-submodules $U$ and $W$.

We continue to assume that $U>0$. Since $U<V$ and $\mathbf{C}_{U}(K)=0$, we can apply the inductive hypothesis with $U$ in place of $V$. We conclude that there exists an element $x \in K t$ such that $\operatorname{dim} \mathbf{C}_{U}(x) \leq(1 / p) \operatorname{dim} U$, where $p$ is the smallest prime divisor of $|G|$. Also $\mathbf{C}_{W}(N)=0$, and we apply the inductive hypothesis with $W$ in place of $V$, and with $N$ and $x$ in place of $K$ and $t$. We obtain an element $y \in N x \subseteq K t$, such that $\operatorname{dim} \mathbf{C}_{W}(y) \leq(1 / p) \operatorname{dim} W$. Now $y=n x$ for some element $n \in N$, and since $n$ centralizes $U$, it follows that $\mathbf{C}_{U}(y)=\mathbf{C}_{U}(x)$. Since $V$ is the direct sum of the $F G$-submodules $U$ and $W$, and the fixed-point-space dimensions of $y$ in each of $U$ and $W$ is at most $(1 / p)$ times the dimension of $U$ and $W$, respectively, it follows that $\operatorname{dim} \mathbf{C}_{V}(y) \leq(1 / p) \operatorname{dim} V$, as required.

What remains is to consider the case where $K$ is a minimal normal subgroup of $G$. Since $G / K$ is cyclic, it follows by Theorem 2.4 that we can write $G=\langle x, y\rangle$, where both $x$ and $y$ lie in $K t$. But $\mathbf{C}_{V}(G)=0$, and thus $\mathbf{C}_{V}(x) \cap \mathbf{C}_{V}(y)=0$. It follows that at least one of $\mathbf{C}_{V}(x)$ or $\mathbf{C}_{V}(y)$ has dimension no greater than $(1 / 2) \operatorname{dim} V$. This completes the proof in the case where $p=2$.

We can now assume $p>2$, and so $G$ is solvable by the Feit-Thompson Theorem. The minimal normal subgroup $K$, therefore, is an elementary abelian $q$-group for some prime $q \geq p$. Also, since $\mathbf{C}_{V}(K)=0$, we know that $q$ is not the characteristic of $F$. We can extend the field $F$ without affecting dimensions of fixed-point spaces, and so it is no loss to assume that $F$ is algebraically closed. (Of course, by Maschke's theorem, this does not affect the complete reducibility of $V$ as an $F K$-module.)

Write $V$ as the direct sum of its "isotypic" components $V_{i}$. In other words, each of the $V_{i}$ is a homogeneous $F K$-submodule of $V$, and if $i \neq j$, then the unique simple component of $V_{i}$ is not isomorphic to the unique simple component of $V_{j}$. These isotypic components are permuted by the action of $G$ on $V$.

Suppose first that none of the isotypic components $V_{i}$ is $\langle t\rangle$-invariant, and consider the subspace $W$ of $V$ obtained by summing the $V_{i}$ in a single $\langle t\rangle$-orbit. (Note that since this is a nontrivial orbit and the size $m$ of this orbit divides $|G|$, it follows that $m \geq p$.) The subspace $W$ is the direct sum of $m$ subspaces of the form $V_{i}$, and these are transitively permuted by $\langle t\rangle$. If $w \in \mathbf{C}_{W}(t)$ has a trivial projection into one of the summands $V_{i}$, then by the transitivity of $\langle t\rangle$ on the summands, it follows that $w$ also has a trivial projection into every other summand of $W$, and so 
$w=0$. In other words, the projection map from $\mathbf{C}_{W}(t)$ into $V_{i}$ is injective, and we have

$$
\operatorname{dim} \mathbf{C}_{W}(t) \leq \operatorname{dim} V_{i}=\frac{1}{m} \operatorname{dim} W \leq \frac{1}{p} \operatorname{dim} W .
$$

Since $V$ is a direct sum of orbit sums like $W$, it follows that $\operatorname{dim} \mathbf{C}_{V}(t) \leq(1 / p) \operatorname{dim} V$, and the proof is complete in this case.

Finally, suppose that one of the isotypic components $V_{i}$ is $\langle t\rangle$-invariant. Since $K$ is abelian, $F$ is algebraically closed and $V_{i}$ is a homogeneous $F K$-module, it follows that each element of $K$ acts via scalar multiplication on $V_{i}$ and thus $[K, t]$ acts trivially on $V_{i}$. But $\mathbf{C}_{V}(K)=0$, and thus $[K, t]<K$. Since $K$ is minimal normal in $G$, and $[K, t] \triangleleft G$, we have $[K, t]=1$, and thus $G$ is abelian. In particular, $|K|=q$, and we can write $K=\langle k\rangle$.

Now let $U_{j}=\mathbf{C}_{V}\left(k^{j} t\right)$ for $0 \leq j \leq q-1$, and note that since $G$ is abelian, each of these subspaces is $G$-invariant. If we can show that $\sum U_{j}$ is direct, it will follow that at least one of the subspaces $U_{j}$ has dimension not exceeding $(1 / q) \operatorname{dim} V$, and since $q \geq p$, the proof will be complete.

Suppose, by way of contradiction, that there exist vectors $x_{j} \in U_{j}$, not all 0 , and such that $\sum x_{j}=0$. Choose these vectors so that as few of them as possible are nonzero, and suppose that $x_{a}$ is nonzero. Apply $k^{a} t$ to the equation $\sum x_{j}=0$, and subtract the result from the original equation. Since $k^{a} t$ fixes $x_{a}$ and stabilizes each of the spaces $U_{j}$, what results is an equation of the form $\sum y_{j}=0$, where $y_{j} \in U_{j}$ and $y_{j}=0$ if $x_{j}=0$. Also, $y_{a}=0$, and by the choice of the $x_{j}$ it follows that all $y_{j}=0$, and thus $k^{a} t$ fixes all $x_{j}$. Now since $\sum x_{j}=0$, there must exist $x_{b} \neq 0$ with $b \neq a$. This vector is fixed by both $k^{a} t$ and $k^{b} t$, and it follows that $x_{b}$ is fixed by $k$. This is a contradiction, since $\mathbf{C}_{V}(K)=0$ and $K=\langle k\rangle$. The proof is now complete.

Recall that the assertion of Corollary B is that

$$
\frac{1}{|G|} \sum_{g \in G} \operatorname{dim} \mathbf{C}_{V}(g) \leq \frac{p+1}{2 p} \operatorname{dim} V,
$$

where $p$ is the smallest prime divisor of $|G|$. (We are assuming here that $V$ is a nonzero, completely reducible $F G$-module and that $\mathbf{C}_{V}(G)=0$.) To prove this we use the following lemma of D. Segal and A. Shalev.

Lemma 2.5 (Segal and Shalev). Let $V$ be an FG-module, where $F$ and $G$ are arbitrary, and let $m$ be the minimum of $\operatorname{dim} \mathbf{C}_{V}(g)$ for $g \in G$. Then

$$
\frac{1}{|G|} \sum_{g \in G} \operatorname{dim} \mathbf{C}_{V}(g) \leq \frac{1}{2}(\operatorname{dim} V+m) .
$$

In our case, we know by Theorem A that the quantity $m$ of Lemma 2.5 is at $\operatorname{most}(1 / p) \operatorname{dim} V$. Corollary B is then immediate.

Next, we show that the fraction $1 / p$ in Theorem A cannot be replaced by any smaller quantity. To do this, we fix a prime $p$ and a positive number $\varepsilon$. We must find a group $G$ with smallest prime divisor $p$, a field $F$ and a completely reducible nonzero $F G$-module $V$ such that $\mathbf{C}_{V}(G)=0$ and

$$
\operatorname{dim} \mathbf{C}_{V}(g)>\left(\frac{1}{p}-\varepsilon\right) \operatorname{dim} V
$$

for each element $g \in G$. 
Let $G$ be an elementary abelian $p$-group of order $p^{d}$ with $d>0$, and let $F$ be any algebraically closed field of characteristic different from $p$. Then there exist $p^{d}-1$ pairwise nonisomorphic nonprincipal one-dimensional $F G$-modules, and we let $V$ be the direct sum of these, so that $\operatorname{dim} V=p^{d}-1$. (Note that $\mathbf{C}_{V}(G)=0$ since the principal module is not a component of $V$.)

If $1 \neq g \in G$, then the direct summands of $V$ with kernel containing $g$ correspond exactly to the $p^{d-1}-1$ nonprincipal one-dimensional modules of $G /\langle g\rangle$. Thus $\operatorname{dim} \mathbf{C}_{V}(g)=p^{d-1}-1$, and it follows that for each nonidentity element $g$ of $G$, we have

$$
\operatorname{dim} \mathbf{C}_{V}(g)=\frac{p^{d-1}-1}{p^{d}-1} \operatorname{dim} V .
$$

Since $\left(p^{d-1}-1\right) /\left(p^{d}-1\right)$ approaches $1 / p$ as $d$ gets large, we see that for each choice of $\varepsilon>0$, a sufficiently large elementary abelian $p$-group provides the desired example. This completes the proof of Theorem A.

Finally, in this example, we compute that

$$
\sum_{g \in G} \operatorname{dim} \mathbf{C}_{V}(g)=\left(p^{d}-1\right)+\left(p^{d}-1\right)\left(p^{d-1}-1\right)=\left(p^{d}-1\right) p^{d-1},
$$

and so

$$
\frac{1}{|G|} \sum_{g \in G} \operatorname{dim} \mathbf{C}_{V}(g)=\frac{1}{p}\left(p^{d}-1\right)=\frac{1}{p} \operatorname{dim} V .
$$

If we take $p=2$, we see that (as we stated in the Introduction) the fraction $3 / 4$ that appears in Corollary B cannot be reduced to a number less than $1 / 2$. We stress, however, that we do not know that $3 / 4$ is the smallest possible coefficient.

\section{Character Degrees AND Class sizes}

In this section, we show the connection between the dimension problem for fixedpoint-spaces and Conjecture C.

Proof of Corollary D. Since $\chi$ is primitive and $G$ is solvable, we can write $\chi=\alpha \beta$, where $\alpha$ is $p$-special and $\beta$ is $p^{\prime}$-special, and each of these characters is primitive. (See, for instance, [4.) Since $\alpha(1)=\chi(1)_{p}$, we can replace $\chi$ by $\alpha$ and assume that $\chi$ is $p$-special. Also, it is clear that we can assume that the $p$-special character $\chi$ is faithful, it follows that $\mathbf{O}_{p^{\prime}}(G)$ is trivial, and hence the Fitting subgroup $\mathbf{F}(G)$ is a p-group. Also, since $G$ has a faithful primitive character, we know that every abelian normal subgroup of $G$ is central and cyclic. Since $G$ is solvable and nonabelian, we see that $\mathbf{F}(G)$ must also be nonabelian, and so it is a central product of the cyclic center of $G$ with an extraspecial p-group $E$, normal in $G$. Also, it is not hard to see that $E / \mathbf{Z}(E)$ is completely reducible as a $G$-module, and we write $V=E / \mathbf{Z}(E)$.

We know that

$$
\chi(1) \leq|E: Z|^{1 / 2}|G: E|_{p} \leq|E: Z|^{3 / 2}=|V|^{3 / 2}
$$

where we have used, for instance, [8] to obtain the second inequality.

On the other hand, we see that $\mathbf{C}_{V}(G)=0$, and it follows from Theorem A that there exists $g \in G$ such that

$$
\operatorname{dim} \mathbf{C}_{V}(g) \leq \frac{1}{2} \operatorname{dim} V
$$


This means that $\left|\mathbf{C}_{V}(g)\right| \leq|V|^{1 / 2}$, and so

$$
\left|\mathrm{cl}_{G}(g)\right|_{p} \geq|V|^{1 / 2} \geq\left(\chi(1)^{2 / 3}\right)^{1 / 2}=\chi(1)^{1 / 3} \text {. }
$$

The result now follows.

\section{REFERENCES}

[1] J. H. Conway, R. T. Curtis, S. P. Norton, R. A. Parker, and R. A. Wilson, Atlas of Finite Groups, Oxford University Press, London, 1984. MR0827219 (88g:20025)

[2] S. Dolfi, Prime factors of conjugacy class lengths and irreducible character degrees in finite soluble groups, J. Algebra 174 (1995), 749-752. MR.1337168 (96c:20011)

[3] R. M. Guralnick and W. M. Kantor, Probabilistic generation of finite simple groups. J. Algebra 234 (2000), no. 2, 743-792. MR:1800754 (2002f:20038)

[4] I. M. IsaACs, Primitive characters, normal subgroups, and $M$-groups, Math. Z. 177 (1981), 267-287. MR0612879 (82f:20026)

[5] P. M. Neumann, A study of some finite permutation groups, Ph.D. Thesis, Oxford, 1966.

[6] P. M. Neumann and M. R. Vaughan-Lee, An essay on BFC-groups, Proc. London Math. Soc. 35 (1977), 213-237. MR0463311 (57:3264)

[7] D. Segal and A. Shalev, On groups with bounded conjugacy classes, Quart. J. Math. Oxford 50 (1999), 505-516. MR.1726791 (2000j:20053)

[8] T. R. Wolf, Sylow $p$-subgroups of $p$-solvable subgroups of GL( $n, p)$, Arch. Math. 43 (1984), 1-10. MR0758331 (86g:20064)

Department of Mathematics, University of Wisconsin, Madison, 480 Lincoln Drive, MADISON, WisCONSIN 53706

E-mail address: isaacs@math.wisc.edu

Department of Mathematics, Texas State University, San Marcos, Texas 78666

E-mail address: tk04@txstate.edu

Department of Mathematics, Michigan State University, East Lansing, Michigan 48824

E-mail address: meier@math.msu.edu

Departament d’Àlgebra, Universitat de València, 46100 Burjassot, ValènCia, Spain

E-mail address: Alexander.Moreto@uv.es 